\title{
Inorganic Acid Catalysed Nitrogen-doped Carbon Dots from Kraft Lignin Waste and their Brain Cell Imaging Applications
}

\author{
Yixian Pei ${ }^{1}$, An-Yi Chang ${ }^{1}$, Xuan Liu ${ }^{1}$, Hua Wang ${ }^{2}$, Hongbo Zhang ${ }^{3}$, Adarsh Radadia ${ }^{1}$, \\ Yuxin Wang ${ }^{1}$, William Yu ${ }^{4}$, and Shengnian Wang $^{1}$ \\ ${ }^{1}$ Louisiana Tech University \\ ${ }^{2}$ Louisiana State University in Shreveport \\ ${ }^{3}$ Louisiana State University Health Sciences Center Shreveport \\ ${ }^{4}$ Louisiana State University Shreveport
}

July 8, 2020

\begin{abstract}
Carbon quantum dots (C-QDs) show great potential to replace traditional semiconductive quantum dots as the next generation of fluorescent probes. We demonstrate here a new C-QD production process using lignin, a high-volume but low market-value industrial waste and/or environmental hazards, as the starting carbon source. By adding a small amount of inorganic acid, the rich phenolic components in lignin were successfully converted to C-QDs through a coking formation mechanism similar to what happens on solid acid catalysts in traditional fossil fuel cracking process. The aqueous solution presence of the received lignin C-QDs is beneficial for brain cell imaging applications, attributing to their fast internalization, low toxicity, tunable photoluminescence by appropriate acidity and reaction temperature during hydrothermal synthesis. This method not only provides a low-cost C-QDs production route, but also helps gain extra profit and/or improve environment for many small agricultural business and paper and pulp industry located in rural area.
\end{abstract}

\section{Hosted file}

Lignin CDs AIChE.doc available at https://authorea.com/users/341054/articles/468021inorganic-acid-catalysed-nitrogen-doped-carbon-dots-from-kraft-lignin-waste-and-theirbrain-cell-imaging-applications 